\title{
MicroRNA-200b and -301 are associated with gemcitabine response as biomarkers in pancreatic carcinoma cells
}

\author{
NAOTAKE FUNAMIZU ${ }^{1}$, CURTIS RAY LACY ${ }^{2}$, MINORI KAMADA ${ }^{3}$, \\ KATSUHIKO YANAGA ${ }^{1}$ and YOSHINOBU MANOME ${ }^{3}$ \\ ${ }^{1}$ Department of Surgery, The Jikei University School of Medicine, Tokyo 105-8461, Japan; \\ ${ }^{2}$ Howard University School of Medicine, Washington DC 20059, USA; \\ ${ }^{3}$ Department of Molecular Cell Biology, The Jikei University School of Medicine, Tokyo 105-8461, Japan
}

Received March 29, 2018; Accepted November 13, 2018

DOI: $10.3892 /$ ijo.2019.4676

\begin{abstract}
Chemotherapy resistance (congenital or acquired) is one of the principal challenges for the treatment of pancreatic carcinoma. Recent evidence has demonstrated that epithelial to mesenchymal transition (EMT) is associated with chemoresistance in pancreatic carcinoma cells. However, the molecular mechanism underlying the development of chemoresistance remains unknown, and limited therapeutic options are available. Therefore, to anticipate individual chemosensitivity or acquired chemoresistance for patients with pancreatic carcinoma, predictive biomarkers are urgently required. Extensive evidence suggests that microRNAs (miRNAs) serve a crucial role in regulating EMT. The aim of this study was to examine the potential role of miRNA (miR)-200b and miR-301 in predicting the chemo-responses to treatment for pancreatic carcinoma. The present results demonstrate that miR-200b expression predicted chemo-sensitivity and may have potential as a biomarker. In six different pancreatic carcinoma cell lines (Capan-1, Capan-2, Panc-1, MIAPaCa-2, BxPC-3 and PL45 cells), the expression of miR-200b correlated positively with chemosensitivity. Moreover, the enhanced expression of miR-200b increased chemosensitivity and induced mesenchymal to epithelial transition. Conversely, miR-301 modulated gemcitabine resistance and induced EMT through the downregulation of cadherin 1 expression. In addition, gemcitabine-resistant cells (Capan-2 and Panc-1) exhibited upregulated miR-301 expression and downregulated gemcitabine-induced apoptosis. In summary, these two miRNAs may serve roles as biomarkers in pancreatic carcinoma, miR-200b expression may predict chemosensitivity, and elevated miR-301 expression may have potential applications in the prediction of acquired gemcitabine resistance.
\end{abstract}

Correspondence to: Dr Naotake Funamizu, Department of Surgery, The Jikei University School of Medicine, 3-25-8 Nishi-shinbashi, Minato-ku, Tokyo 105-8461, Japan

E-mail: funamizujikei@yahoo.co.jp

Key words: pancreatic cancer, microRNA-301, microRNA-200b, gemcitabine resistance

\section{Introduction}

Pancreatic carcinoma is one of the most lethal diseases, with an extremely poor prognosis. Current cancer statistics indicate that pancreatic carcinoma is the fourth leading cause of cancer-associated mortality in the USA, with an incidence of 53,000 new cases and a high mortality rate of 42,000 in 2016 (1). For patients with unresectable or recurrent pancreatic carcinoma, the standard chemotherapy is gemcitabine in combination with other chemo-therapeutic agents (2). Despite accumulated knowledge regarding pancreatic carcinoma etiology, the prognosis has not significantly improved in the last decade (3). The development of gemcitabine resistance during chemotherapy serves an important role in the prognosis of pancreatic carcinoma and has become an increasingly common phenomenon (4). However, the molecular mechanisms underlying gemcitabine resistance remain unclear. Therefore, to improve the prognosis of patients with pancreatic carcinoma, it is important to identify innovative biomarkers that are able to predict the risk of recurrence and chemoresistance in patients who are receiving gemcitabine-based chemotherapy.

MicroRNAs (miRNAs) represent novel single-stranded, small non-coding RNA molecules. Mature miRNAs bind directly to specific targets within mRNA 3'-untranslated regions of target RNAs and negatively regulate translation or mRNA cleavage through partial sequence homology at the post-transcriptional level (5). Previous data have demonstrated frequent deregulation of miRNAs in the majority of malignant human tumors (6-9). Deregulated miRNAs are associated with behavior as either oncogenes or tumor suppressor genes. Certain miRNAs have been implicated in cellular processes involving proliferation, invasiveness, apoptosis, and chemoresistance (10-13). Moreover, current evidence has demonstrated that miRNAs are critically involved in regulating drug resistance-mediated epithelial to mesenchymal transition (EMT) (14-16).

Among these miRNAs, there is evidence that miRNA (miR)-200b is downregulated in numerous types of cancer, including pancreatic, colorectal, gastric and lung cancer (17-22). Additionally, miR-200b inhibition induces EMT through upregulated zinc finger E-box-binding homeobox 1 (ZEB1) (23) and induces chemoresistance $(24,25)$. 
By contrast, miR-301 has been reported to be associated with cell invasion, migration and drug-resistance in breast cancer(26). More recently, data have demonstrated that miR-301 is associated with gemcitabine resistance through EMT and enhanced cell proliferation in pancreatic carcinoma (13). Based on these reports, the purpose of the present study was to investigate the roles of miR-200b and miR-301 as potential biomarkers for chemosensitivity or acquired chemoresistance in pancreatic carcinoma. A total of six different pancreatic carcinoma cell lines were used to investigate the mechanisms of miR-200b and miR-301 expression in pancreatic carcinoma. It was observed that the miR-200b expression level correlated with cadherin 1 (CDH1) expression and chemosensitivity in the six cell lines. In addition, it was demonstrated that transfection with miR-200b upregulated gemcitabine sensitivity. Conversely, it was observed that stable gemcitabine-resistant cell lines, Capan-2 and Panc-1, exhibited increased miR-301 expression and inhibited CDH1 expression. Unlike miR-200b, the overexpression of miR-301 induced chemoresistance and reduced apoptosis. These findings indicated that the miR-200b/CDH1 and miR-301/CDH1 signaling axes serve important roles in mediating the response to chemotherapy in pancreatic carcinoma (Fig. 1). Moreover, these results implied that miR-200b and miR-301 may be potential therapeutic targets in pancreatic carcinoma.

\section{Materials and methods}

Oligonucleotides. Pre-miR-200b (cat.no.PM10492), pre-miR-301 (cat. no. PM12929), negative control (cat. no. AM17110), miR-200b inhibitor (cat. no. AM10492), miR-301 inhibitor (cat. no. AM12929) and their negative controls (cat. no. AM17010) were purchased from Ambion (Thermo Fisher Scientific, Inc., Waltham, MA, USA).

Cell culture conditions. Human pancreatic carcinoma cell lines (Capan-1, Capan-2, Panc-1, MIAPaCa-2, BxPC-3 and PL45) were obtained from the American Type Culture Collection (Manassas, VA, USA). Panc-1, MIAPaCa-2 and PL45 were maintained in Dulbecco's modified Eagle's medium (DMEM; Thermo Fisher Scientific, Inc.; cat. no. 10566-016) supplemented with $10 \%$ fetal bovine serum (FBS). Capan-1, Capan-2 and BxPC-3 cells were grown in RPMI-1640 (cat. no. 61870-036) with 10\% FBS (both from Thermo Fisher Scientific, Inc.; cat. no. 10437028). The two media contained antibiotics (100 U/ml penicillin and $100 \mu \mathrm{g} / \mathrm{ml}$ streptomycin). All cell lines were routinely passaged as monolayer cultures at $37^{\circ} \mathrm{C}$ in a humidified atmosphere of $95 \%$ air and $5 \% \mathrm{CO}_{2}$.

Establishment and characterization of gemcitabine-resistant Panc-1 and Capan-2 cells. In order to further identify the association between chemoresistance and miRNA expression, gemcitabine-resistant cells were generated. The gemcitabine-resistant cell lines (Capan-2 GEM-R and Panc-1 GEM-R) were generated in RPMI-1640 or DMEM medium with $15 \%$ FBS and continuous exposure to the respective half-maximal inhibitory concentration $\left(\mathrm{IC}_{50}\right)$ of gemcitabine $(\mu \mathrm{M})$ (Tocris Bioscience, Bristol, UK) for $>5$ months (27). To assess the gemcitabine resistance of Capan-2 GEM-R and Panc-1 GEM-R cells, colorimetric assays were performed following treatment with gemcitabine. Secondly, in the two generated gemcitabine resistant cell lines, miR-301 was measured by reverse transcription-quantitative polymerase chain reaction.

$R N A$ preparation and $R T-q P C R$ analysis. RT-qPCR was performed as described previously (28). Total RNA was extracted from cultured cells using a standard TRIzol ${ }^{\circledR}$ (Invitrogen; Thermo Fisher Scientific, Inc.) protocol. Cell pellets were suspended in an aliquot of $1 \mathrm{ml} /$ well of TRIzol in a 6-well plate. Isolated RNA was reverse-transcribed using a High-Capacity cDNA RT kit (Applied Biosystems; Thermo Fisher Scientific, Inc.), according to the manufacturer's protocol. cDNA was diluted and stored at $-20^{\circ} \mathrm{C}$ prior to use. Gene expression levels were measured with a custom-designed, TaqMan qPCR (Applied Biosystems; Thermo Fisher Scientific, Inc.) containing probes for six genes: CDH1 (ID: Hs00156401_m1), ZEB1 (ID: Hs00232783_m1), ZEB2 (ID: Hs00207691_m1), Vimentin (ID: Hs00185584_m1), interleukin (IL)-6 (ID: Hs00174131_m1), miR-200b (ID: 002251) and miR-301 (ID: 002392), with GAPDH (ID: Hs99999901_s1) for mRNA or RNAU6 (ID: 001002) for miRNA as an internal control. The relative expression levels of genes, miR-200b and miR-301 relative to GAPDH or RNAU6 were calculated using the relative quantification $\Delta \Delta \mathrm{Cq}$ method (29). RT-qPCR reactions were performed using TaqMan Gene Expression Assays on an ABI prism 7900HT Sequence Detection instrument (Applied Biosystems; Thermo Fisher Scientific, Inc.). qPCR was performed as follows: $95^{\circ} \mathrm{C}$ for $10 \mathrm{~min}$, followed by 40 cycles of $95^{\circ} \mathrm{C}$ for $15 \mathrm{sec}$ and $60^{\circ} \mathrm{C}$ for $60 \mathrm{sec}$, according to the manufacturer's protocol. Each sample was assayed in triplicate.

Pre-miR-200b, miR-200b and miR-301 inhibitor transfection experiments. miRNA precursor molecules corresponding to miR-200b or miR-200b inhibitor, and miR-301 inhibitor, were transfected using the RNAiMAX Transfection Reagent (Invitrogen; Thermo Fisher Scientific, Inc.) into Capan-2 and Panc- 1 cells, and the effects on the respective oligonucleotide were measured by RT-qPCR. Capan- 2 and Panc- 1 cells at a density of $1 \times 10^{5}$ cells/well were transfected with $50 \mathrm{nM}$ microRNA in a 6-well plate for RNA extraction or a $10-\mathrm{cm}$ dish for gemcitabine sensitivity and proliferation assays, following the manufacturer's protocol. Cells in the 6-well plate were collected $48 \mathrm{~h}$ post-transfection to extract RNA and measured for miR-200b or miR-301 expression. After $12 \mathrm{~h}$ of transfection, transfected cells in the $10-\mathrm{cm}$ dish were seeded into 96 -well plates for proliferation. These transfection experiments were repeated independently three times.

Gemcitabine sensitivity assay with transfection of pre-miR-200b or miR-200b and miR-301 inhibitors. The colorimetric assay was performed essentially as described in a previous method (30). Briefly, cells were seeded in a $6-\mathrm{cm}$ dish at $70 \%$ confluency. After $12 \mathrm{~h}$, pre-miR-200b or miR-200b/301 inhibitors and respective controls were transfected in each dish overnight. Transfected cells were seeded in 96-well plates at 4,000 cells/well in triplicate. After incubating for $12 \mathrm{~h}$, cell viability was determined by treating the cells with stepwise 4-fold serial dilutions of gemcitabine (from $100 \mu \mathrm{M}$ ) and incubated at $37^{\circ} \mathrm{C}$ for $96 \mathrm{~h}$. To evaluate cell survival, the cells were fixed with $25 \%$ glutaraldehyde for $30 \mathrm{~min}$ at room temperature and stained with $200 \mu 10.05 \%$ methylene blue for 


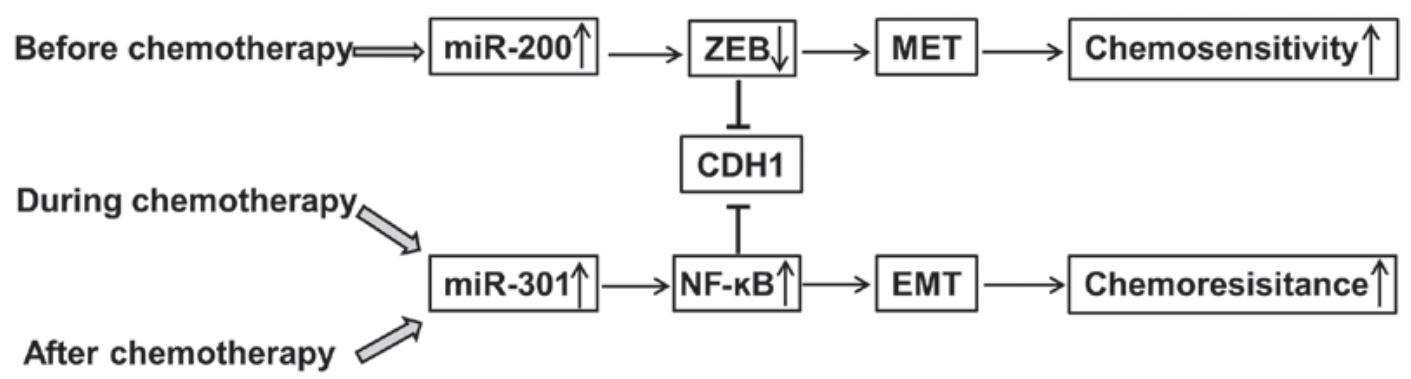

Figure 1. A putative pathway for predicting chemosensitivity and chemoresistance. The proposed pathway demonstrates that miR-200b and miR-301 may control chemosensitivity or resistance through EMT. EMT, epithelial to mesenchymal transition; MET, mesenchymal to epithelial transition; miR, microRNA;

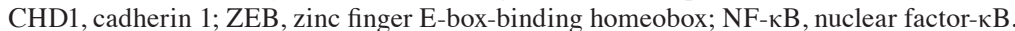

$20 \mathrm{~min}$ at room temperature. The dye was eluted with $0.33 \mathrm{M}$ $\mathrm{HCl}$ for $20 \mathrm{~min}$ with agitation. Absorbance was measured using a microplate reader (model no. 3550; Bio-Rad Laboratories, Inc., Hercules, CA, USA) at $598 \mathrm{~nm}$. The $\mathrm{IC}_{50}$ for cell growth was calculated. The morphology of Panc-1 and Capan-2 cells was assessed using light microscopy (x20 magnification).

Cell proliferation assay. The cell growth of pancreatic carcinoma cell lines was studied using the colorimetric methylene blue assay, as described previously $(31,32)$. To test cell growth, cells were transfected with miR-200b or $200 \mathrm{~b}$ inhibitor or negative control in a $10-\mathrm{cm}$ dish, counting the first $12 \mathrm{~h}$ as Day 0. Transfected cells at 4,000 cells/well were plated in a 96-well plate for $24 \mathrm{~h}$. Mean values were calculated from three different wells in triplicate for 4 days.

Apoptosis assay. To evaluate whether miR-200b or acquired gemcitabine resistance contributes to a decrease in caspase-3/7, Capan-2 or Panc-1 cells were cultured for $12 \mathrm{~h}$ in 96-well plates in triplicate, and treated with $50 \mathrm{nM}$ pre-miR-200b or miR-200b inhibitor, or their respective controls. The assay was analyzed using a caspase-3/7 assay kit (Promega Corporation, Madison, WI, USA), according to the manufacturer's protocol.

Cell invasion assay. The invasion assay was performed in 24-well Biocoat Matrigel invasion chambers (BD Biosciences, San Jose, CA, USA), according to a previous protocol $(25,33)$. Briefly, cells were transfected with pre-miR-200b or pre-miR-301 and the negative control in a $10-\mathrm{cm}$ dish. After $12 \mathrm{~h}$ transfection, cells were harvested and plated in the Matrigel-coated wells ( $4 \times 10^{4}$ cells/well) and control insert wells (4X10 ${ }^{4}$ cells/well) using Capan- 2 and Panc- 1 cells, respectively. After $20 \mathrm{~h}$ incubation, seeded cells on the membrane were removed by wiping with a cotton swab, and the invasive cells through the membrane were fixed with methanol for $5 \mathrm{~min}$ and stained with crystal violet for $5 \mathrm{~min}$. Under a light microscope (x20 magnification), invasive cells were counted in three random fields. All assays were performed in triplicate.

Immunofluorescence imaging. Immunofluorescence was performed as previously described (13). Briefly, cells were seeded into a chamber slide at $40 \%$ confluence. Following incubation overnight, the cells were fixed with $4 \%$ paraformaldehyde for $20 \mathrm{~min}$ at room temperature and permeabilized with $0.15 \%$ Triton X-100 in PBS for 20 min. Subsequently, the cells were blocked with $5 \%$ goat serum (cat. no. ab7481;
Abcam, Cambridge, MA, USA) in PBS for $1 \mathrm{~h}$ at room temperature. $\mathrm{CDH} 1$ and nuclear factor $(\mathrm{NF})-\kappa \mathrm{B}$ protein expression was detected using anti-CDH1 (cat. no. ab15148; Abcam; 1:1,000) and anti-NF- $\kappa$ B (cat. no. 3033; Cell Signaling Technology Inc., Danvers, MA, USA; 1:500) antibodies at $4^{\circ} \mathrm{C}$ overnight, according to the manufacturer's protocol. An Alexa Fluor-conjugated antibody (cat. no. ab1500083; $1: 2,000$ ) was used as a secondary antibody at $37^{\circ} \mathrm{C}$ for $2 \mathrm{~h}$. The cell nucleus was counterstained with DAPI for $20 \mathrm{~min}$ at room temperature. The coverslips were mounted on slides prior to viewing using a fluorescence microscope (magnification, $\mathrm{x} 20)$.

Statistical analysis. All experiments were performed in triplicate and conducted at least twice. Data are presented as the mean \pm standard deviation where applicable. GraphPad Prism version 5.0 (GraphPad Software Inc., La Jolla, CA, USA) was used for all statistical analysis. Levels of significance for comparisons between cell lines were determined by the Student's t-test distribution. To assess the correlation between miR-200b expression, CDH1 expression and $\mathrm{IC}_{50}$, Pearson's correlation analysis was performed in six different pancreatic carcinoma cell lines. To analyze multiple comparisons, one-way analysis of variance and the Bonferroni test as a post hoc test was used. $\mathrm{P}<0.05$ was considered to indicate a statistically significant difference.

\section{Results}

miR-200b expression correlates negatively with the $I C_{50}$ of gemcitabine in pancreatic carcinoma cell lines. To examine the correlation between miR-200b and $\mathrm{IC}_{50}$, the present study focused on $\mathrm{CDH} 1$ expression, which is known to be a target for miR-200b and the EMT markers. To identify the correlation, miR-200b and CDH1 expression was initially investigated using six different cell lines (Capan-1, Capan-2, Panc-1, MIAPaCa-2, BxPC-3 and PL45) using RT-qPCR (Fig. 2A). Next, the $\mathrm{IC}_{50}$ of gemcitabine was measured using a colorimetric assay in the six pancreatic carcinoma cell lines (Fig. 2B). As expected, the results demonstrated a clear positive correlation between miR-200b and CDH1 expression according to the Pearson data $\left(r^{2}=0.8165\right.$; Fig. 2C). By contrast, an inverse correlation between $\mathrm{IC}_{50}$ and miR-200b or CDH1 was observed $\left(\mathrm{r}^{2}=0.7042\right.$ and $r^{2}=0.9030$, respectively; Fig. 2C). These data indicated that miR-200b is a putative biomarker for chemosensitivity in pancreatic carcinoma. 
A

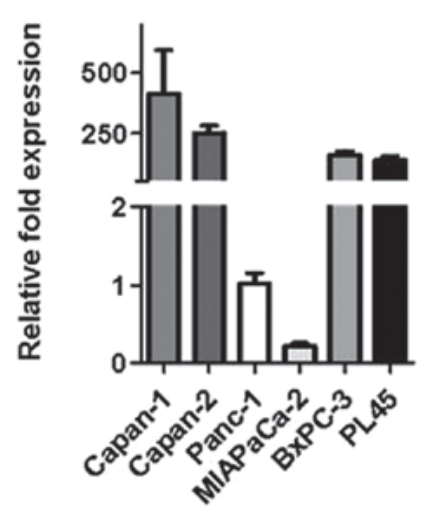

CDH1 expression
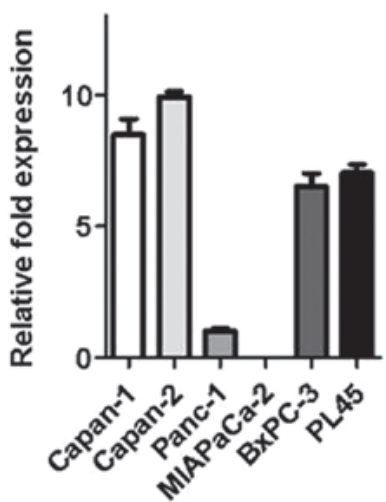

B

Gemcitabine sensitivity assay

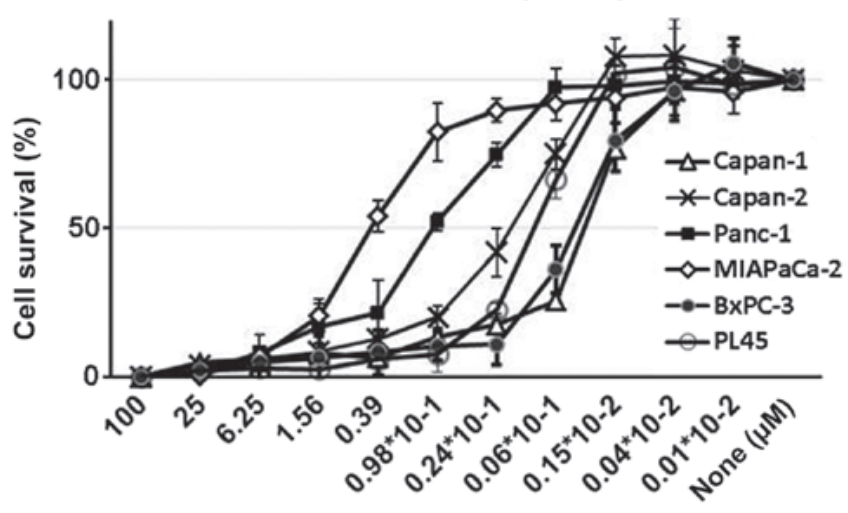

C
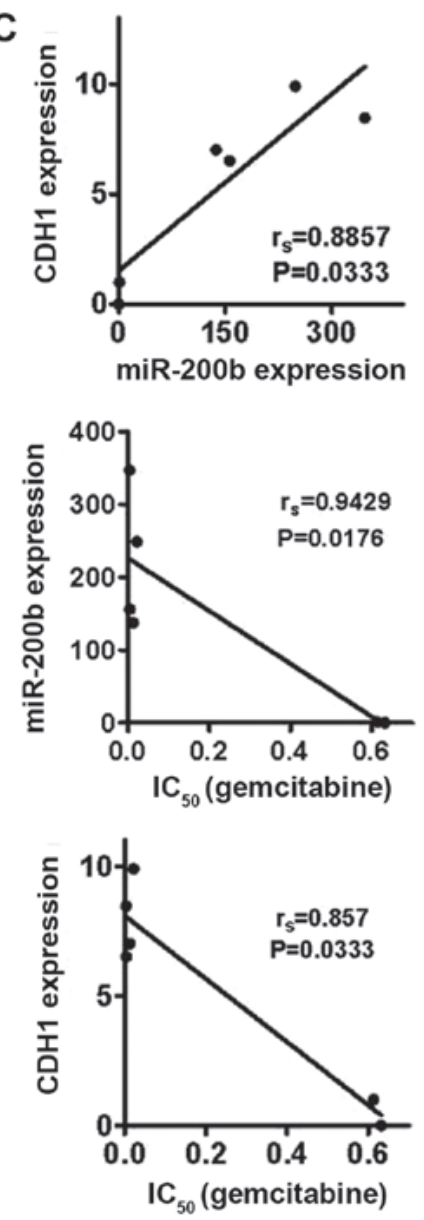

Figure 2. Functional analysis of miR-200b in pancreatic carcinoma cells. (A) miR-200b and CDH1 mRNA were measured using reverse transcription-quantitative polymerase chain reaction analysis in six different pancreatic carcinoma cell lines (Capan-1, Capan-2, Panc-1, MIAPaCa-2, BxPC-3 and PL45). Data represent the mean of triplicate samples from three independent experiments. (B) A gemcitabine sensitivity assay was performed using a colorimetric assay at the $\mathrm{IC}_{50}$ of the cell lines. (C) The correlation between miR-200b and $\mathrm{IC}_{50}$ was evaluated using Pearson's correlation analysis. miR, microRNA; CDH1, cadherin $1 ; \mathrm{IC}_{50}$, half-maximal inhibitory concentration.

Forced expression of miR-200b induces CDH1 expression and promotes gemcitabine sensitivity in Capan-2 and Panc-1 cells. To evaluate the functional role of miR-200b in pancreatic carcinoma, pre-miR-200b or miR-200b inhibitor were transfected into Capan-2 and Panc-1 cells using RNAiMAX Transfection Reagent. The efficacy of the transfection was confirmed using RT-qPCR. miR-200b overexpression upregulated CDH1, and suppressed ZEB1 and ZEB2 (Fig. 3A). Conversely, miR-200b inhibition reduced CDH1 expression in the two cell lines (Fig. 3B). Moreover, miR-200b overexpression improved sensitivity to gemcitabine, and miR-200b inhibitor significantly affected gemcitabine sensitivity in the two cell lines (Fig. 4). Furthermore, the capacity for cell growth was evaluated using a proliferation assay in the two cell types. Forced expression of miR-200b reduced cell growth, while miR-200b inhibition did not affect cell growth (Fig. 5A). These results were consistent across the two cell lines were in accordance with a previous study (25).

miR-200b and miR-301 expression affects apoptosis and cell invasiveness. Overexpressed miR-200b enhanced apoptosis and inhibited cell invasiveness in the two cell lines (Fig. 5B and C). By contrast, downregulated miR-200b reduced apoptosis and enhanced cell invasiveness (Fig. 5B and C).
Gemcitabine-resistant cells were generated from Capan-2 and Panc-1 cells. To examine the mechanisms underlying gemcitabine resistance, two sub-cell lines were generated, which were derived from Capan-2 and Panc-1 cells. These were cultured in the presence of high concentrations of gemcitabine $\left(\mathrm{IC}_{50}\right)$, by gradually increasing the concentration of gemcitabine over 5 months. Cells in which the $\mathrm{IC}_{50}$ values were over four times higher compared with their controls were defined as Capan-2 GEM-R and Panc-1 GEM-R. The $\mathrm{IC}_{50}$ of these cells was evaluated using a colorimetric assay, and they exhibited strong gemcitabine resistance compared with each control cell line (Fig. 6A). Moreover, the two GEM-R cell lines exhibited increased cell growth (Fig. 6B).

Gemcitabine-resistant Capan-2 and Panc-1 cells exhibited increased miR-301 expression. The present study also examined whether acquired gemcitabine resistance in cells was associated with miR-301 expression. Gemcitabine-resistant cells exhibited increased miR-301 and reduced miR-200b expression (Fig. 7A). No explanation became clear as to why miR-200b was reduced in gemcitabine-resistant cells. However, Wang et al (34) recently reported that gemcitabine treatment induced reduced $\mathrm{miR}-200 \mathrm{~b}$ in pancreatic carcinoma cell lines. In addition, resistant cells exhibited an anti-apoptotic 
A miR-200b expression Capan-2

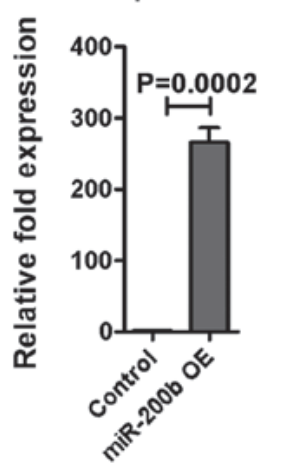

B miR-200b expression Capan-2

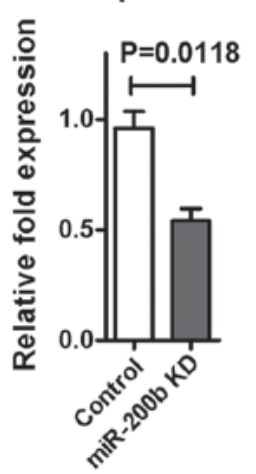

EMT genes expression

Capan-2

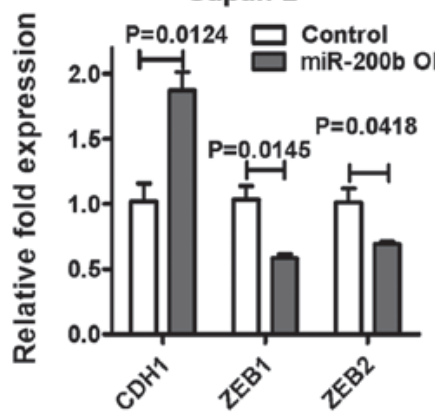

EMT genes expression

Capan-2

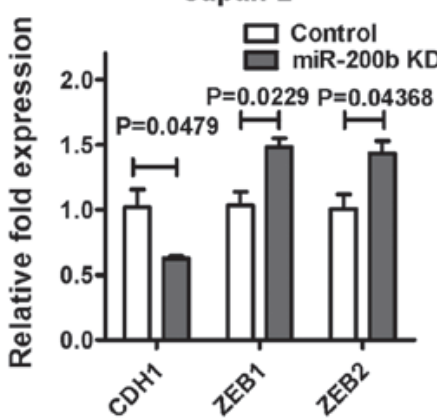

miR-200b expression

Panc-1

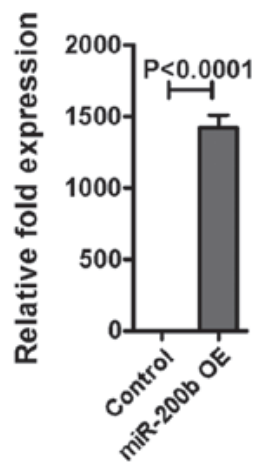

miR-200b expression Panc-1

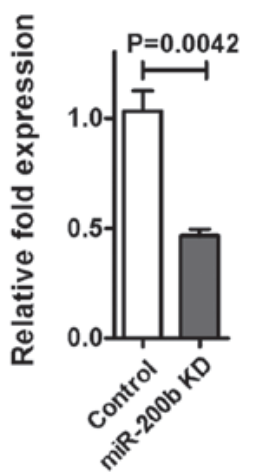

EMT genes expression Panc-1

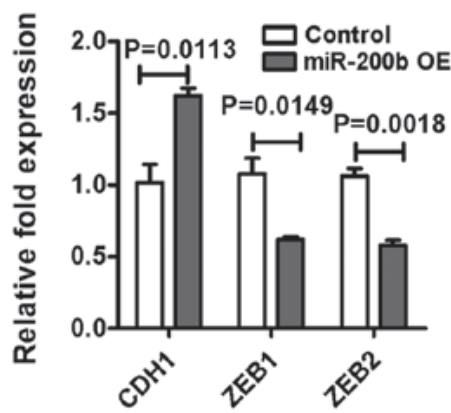

EMT genes expression

Panc-1

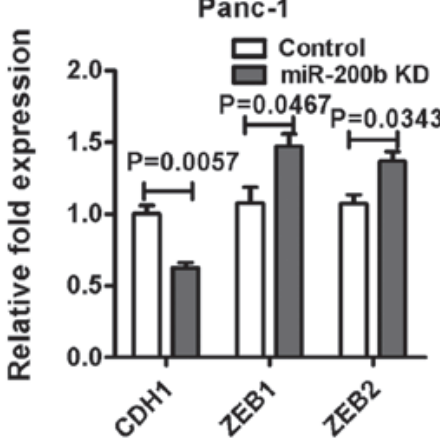

Figure 3. A validation study of the association between miR-200b and EMT-associated genes in pancreatic carcinoma cells. (A) miR-200b was transfected in Capan-2 and Panc-1 cells. Transfection efficiency was determined using reverse transcription-quantitative polymerase chain reaction analysis and is displayed as a relative fold change. Data represent the mean of triplicate samples from three independent experiments. (B) Cells transfected with miR-200b inhibitor exhibited reduced CDH1 expression at the mRNA level in the two cell lines. miR, microRNA; EMT, epithelial to mesenchymal transition; OE, overexpression; ZEB, zinc finger E-box-binding homeobox; CDH1, cadherin 1; KD, knockdown.
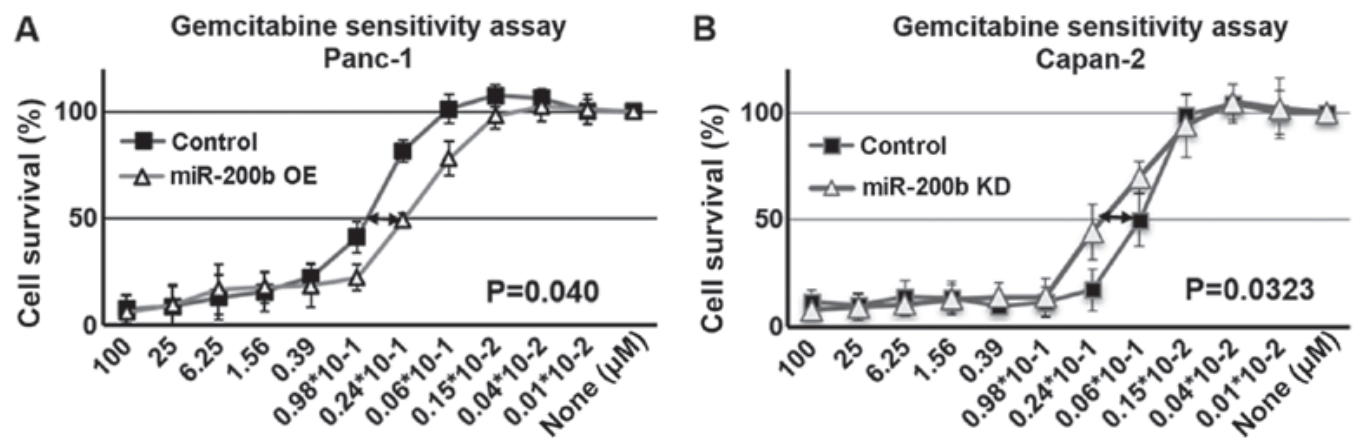

Figure 4. A functional study of the effect of miR-200b on chemosensitivity. (A) miR-200b or its control was transfected at $50 \mathrm{nM}$ for reverse transcription-quantitative polymerase chain reaction analysis in Capan-2 and Panc-1 cells. Transfected cells (miR-200b OE) exhibited increased gemcitabine sensitivity in the two cell lines. All quantitative values are presented as the mean \pm standard deviation. (B) miR-200b inhibition (miR-200b KD) reduced gemcitabine sensitivity in Capan-2 and Panc-1 cells. miR, microRNA; OE, overexpression; KD, knockdown.

effect and increased cell invasiveness compared with control cells (Fig. 7B).

Gemcitabine-resistant cells revealed activated $N F-\kappa B$ and decreased $C D H 1$ expression at the protein level. In the two gemcitabine-resistant cell lines, immunofluorescence was performed for NF- $\mathrm{BB}$ and $\mathrm{CDH} 1$. Gemcitabine-resistant cells exhibited $\mathrm{NF}-\kappa \mathrm{B}$ activation and decreased $\mathrm{CDH} 1$ expression, as expected (Fig. 8A). The difference was less marked in Panc-1 cells due to the lower expression levels of CHD1 and higher activation levels of $\mathrm{NF}-\kappa \mathrm{B}$ in the control cells. It was not possible within the scope of this investigation to perform western blot analysis using a fractionated sample (Cyto/Nuc) and quantification of the expression of target proteins of NF- $\kappa \mathrm{B}$. As an alternative, IL-6 expression was measured to verify the NF- $\kappa \mathrm{B}$ activation in gemcitabine-resistant cells (Fig. 8B). In addition, RT-qPCR analysis demonstrated a decrease in CDH1 expression. The expression of EMT-associated genes, including vimentin, ZEB1 and ZEB2, was measured using RT-qPCR. The results did not exhibit a consistent pattern 
A
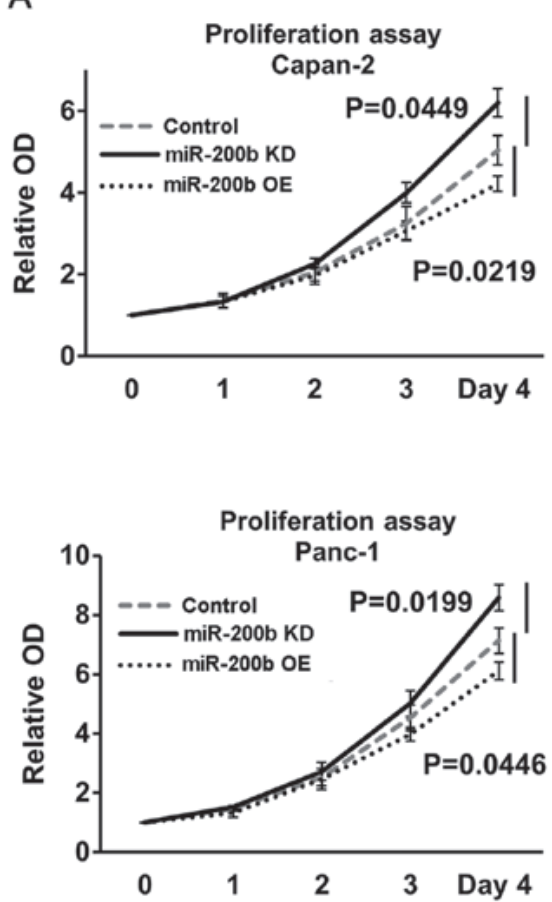

B
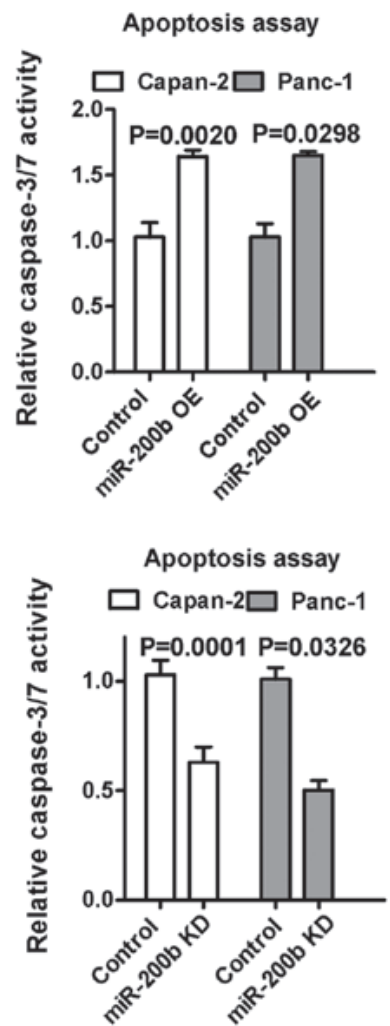

C Invasion assay
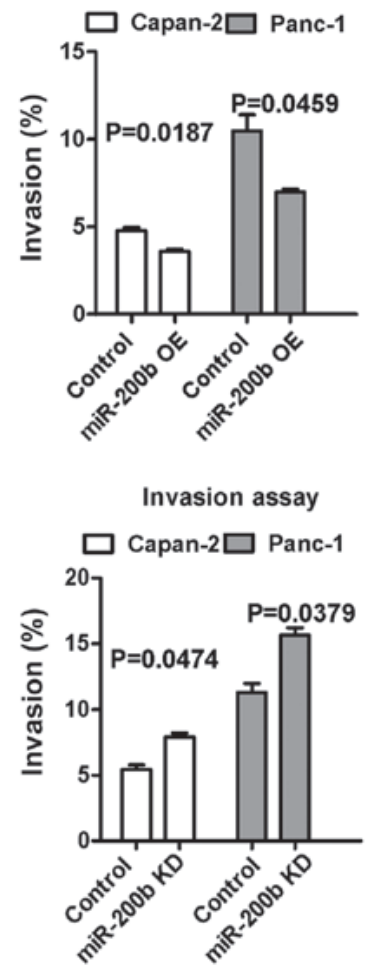

Figure 5. Further analysis of the effect of miR-200b on cell proliferation, apoptosis and invasion. (A) According to the colorimetric assay, miR-200b significantly reduced cell proliferation in Capan-2 and Panc-1 cell lines compared with the control. By contrast, miR-200b inhibition induced cell growth in the two cell lines. Values are the mean of three independent experiments performed in triplicate. Error bars indicate the standard deviation. Data from Day 4 were used for the statistical analysis. (B) Capan-2 or Panc-1 cells were seeded in 96-well plates having been transfected overnight with $50 \mathrm{nM} \mathrm{miR-200b} \mathrm{(miR-200b} \mathrm{OE)}$ or miR-200b inhibitor (miR-200b KD), or their controls. miR-200b OE contributed to the induction of apoptosis. Successful induction of apoptosis was assessed by measuring caspase-3/7 activity. Data represent the mean of three replicates \pm standard deviation. (C) Following transfection with $50 \mathrm{nM}$ miR-200b (miR-200b OE) or miR-200b inhibitor (miR-200b KD), or their controls, cells were seeded in Matrigel-coated chambers for the invasion assays. miR-200b OE significantly reduced cell invasiveness in the two cell lines. Invasive cells were fixed with methanol prior to being stained with crystal violet and photographed. Stained cells were counted in three separate microscopic fields per well. The values were averaged, and the mean \pm standard deviation was calculated from triplicate samples. miR, microRNA; OE, overexpression; KD, knockdown; OD, optical density.

A

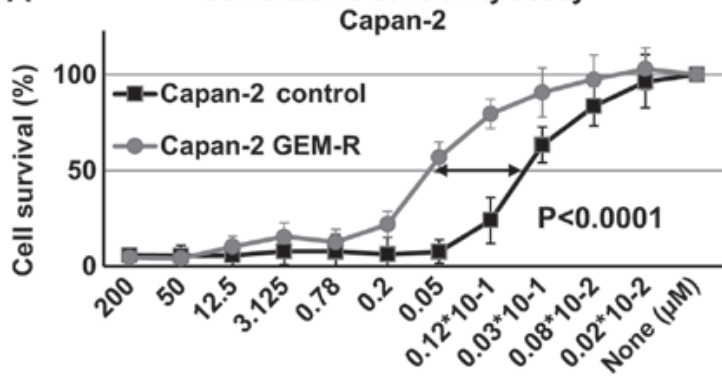

Gemcitabine sensitivity assay

Panc-1

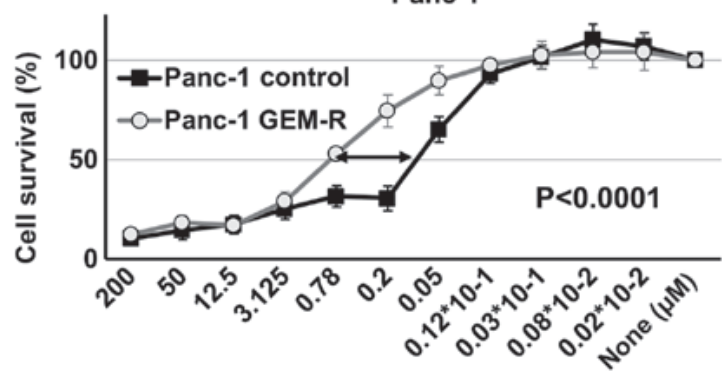

B Proliferation assay

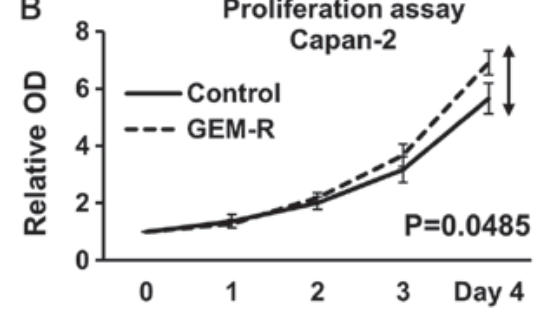

Proliferation assay

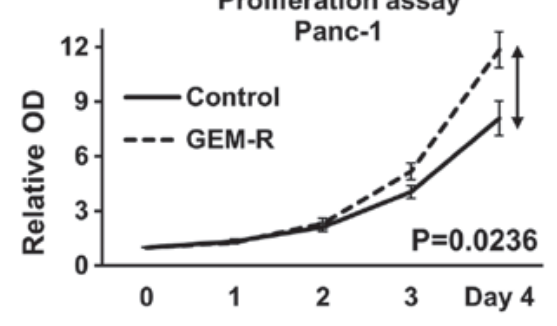

Figure 6. Functional analysis of GEM-R cells. (A) A gemcitabine sensitivity assay was performed using a colorimetric assay. The IC ${ }_{50}$ of the respective GEM-R cells revealed significant resistance to gemcitabine. (B) Cell growth of GEM-R cells was apparently promoted in the two cell lines. GEM-R, gemcitabine-resistant; OD, optical density; $\mathrm{IC}_{50}$, half-maximal inhibitory concentration. 

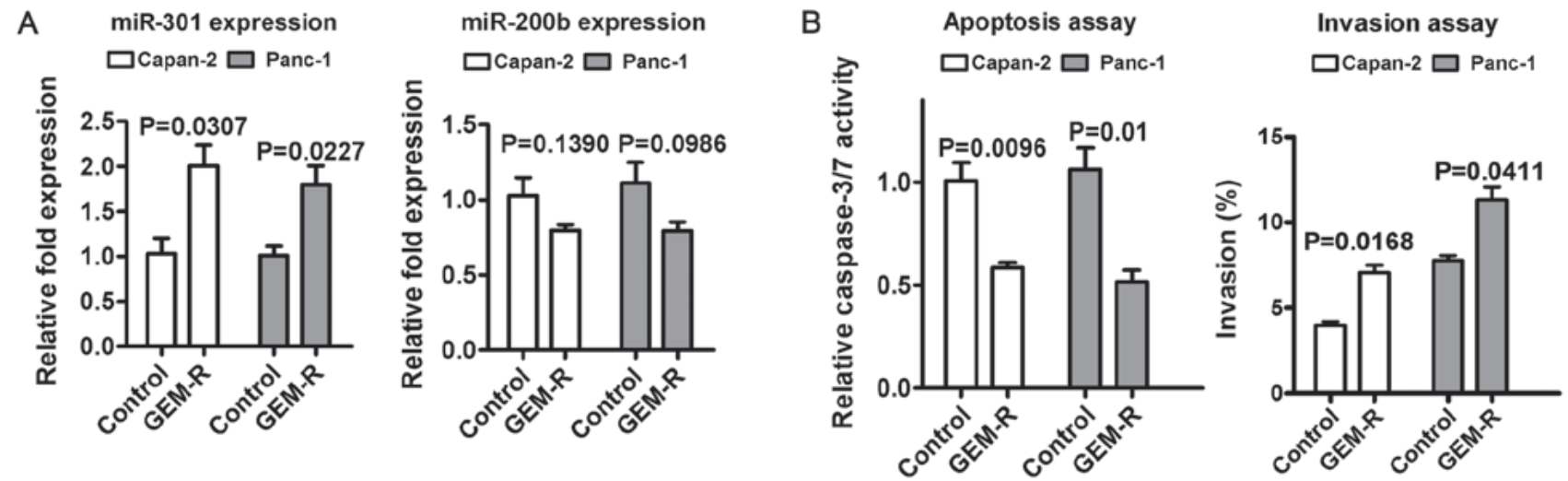

Figure 7. Functional analysis of GEM-R cells regarding miR-301 expression, apoptosis and invasion. (A) GEM-R cells expressed higher levels of miR-301 compared with control Capan-2 and Panc-1 cells. (B) GEM-R cells exhibited resistance to apoptosis and acquired cell invasiveness in the two cell lines. GEM-R, gemcitabine-resistant; miR, microRNA.

A Capan-2
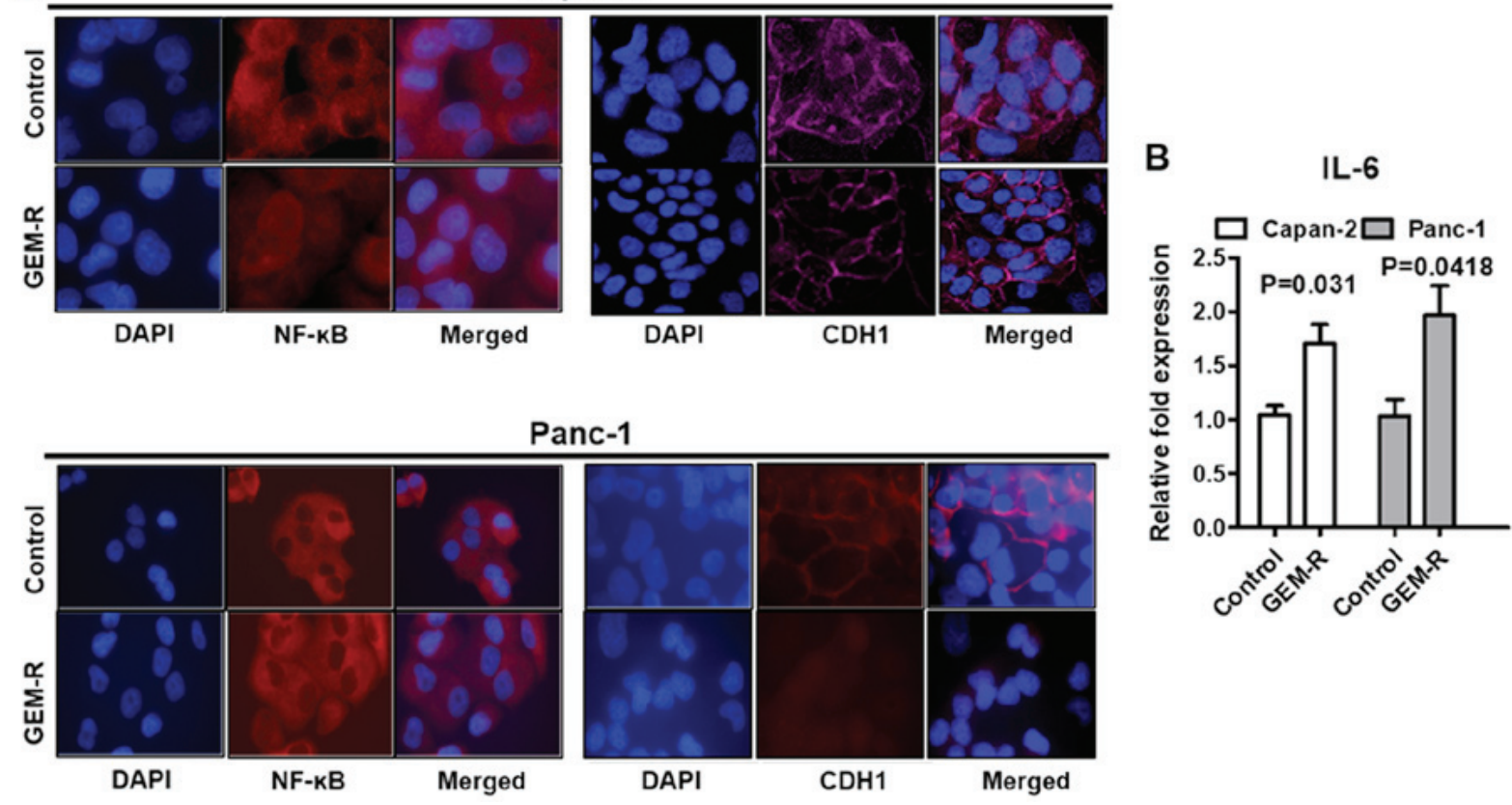

Figure 8. Immunostaining results suggest that GEM-R cells may be involved in NF- $\kappa$ B activity and CDH1 expression in Capan-1 and Panc-1 cell lines (A) GEM-R cells exhibited increased nuclear translocation of p65 compared with control cells in the two cell lines. In addition, GEM-R cells exhibited downregulation of $\mathrm{CDH} 1$ expression. Representative images of two independent experiments are shown (magnification, $\mathrm{x} 20$ ). (B) NF- $\kappa \mathrm{B}$ target gene IL- 6 expression

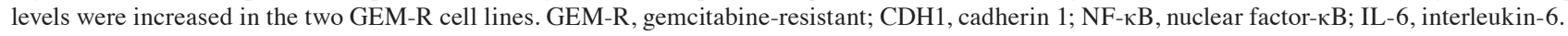

of gene expression to explain how gemcitabine resistance led to EMT in the cell lines (Fig. 9), although western blot analysis was not presented. In addition, morphology remained unaltered in gemcitabine-resistant cells (data not shown). In the same manner, miR-301 overexpression revealed decreased CDH1 expression levels, an enhanced anti-apoptotic effect, an increased $\mathrm{IC}_{50}$ of gemcitabine and increased cell invasiveness, as previously reported (13). Contrary to expectations, miR-301 inhibition did not induce the remission of gemcitabine resistance (Fig. 10). These data are consistent with a previous report (13). Based on the present data, elevated miR-301 expression may affect acquired gemcitabine resistance, and an increase in miR-301 may be a predictive biomarker for acquired gemcitabine resistance in patients with pancreatic carcinoma.

\section{Discussion}

Pancreatic carcinoma has an extremely poor prognosis; early diagnosis is difficult to achieve, and it exhibits aggressive invasion, early distant metastasis, and resistance to anticancer drugs $(1,35)$. However, gemcitabine-based chemotherapy remains the first-line chemotherapy regimen (36). Gemcitabine became the most widely used anticancer drug for the initial treatment of advanced and recurrent pancreatic carcinoma when Burris et al (37) reported in 1997 that gemcitabine had better clinical outcomes compared with 5-fluorouracil. Gemcitabine contributed only 6.2 months to the median survival time for locally advanced or metastatic pancreatic carcinoma (36). Recently, a number of trials have been performed to compare gemcitabine alone with combinations 

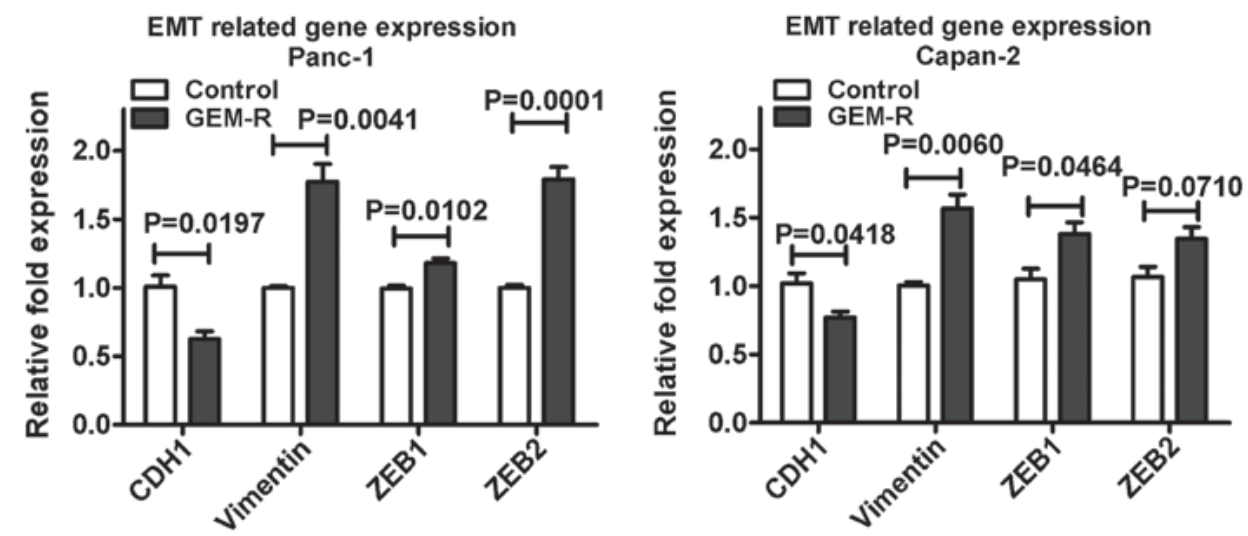

Figure 9. GEM-R cells are associated with EMT. EMT-associated genes, including vimentin, ZEB1 and ZEB2, were quantified using RT-qPCR analysis, and data are expressed as a relative fold change. RT-qPCR data represent the mean of three biological replicates and are representative of three independent experiments. RT-qPCR, reverse transcription-quantitative polymerase chain reaction; GEM-R, gemcitabine-resistant; EMT, epithelial to mesenchymal transition; ZEB, zinc finger E-box-binding homeobox; CDH1, cadherin 1.

miR-301 expression

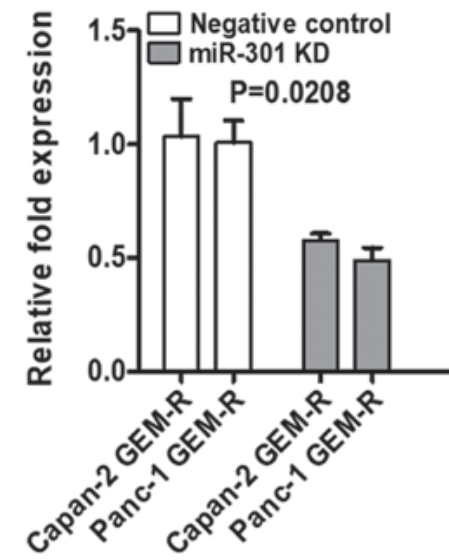

\section{Gemcitabine sensitivity} Capan-2 GEM-R $(0.01 \mu \mathrm{M})$

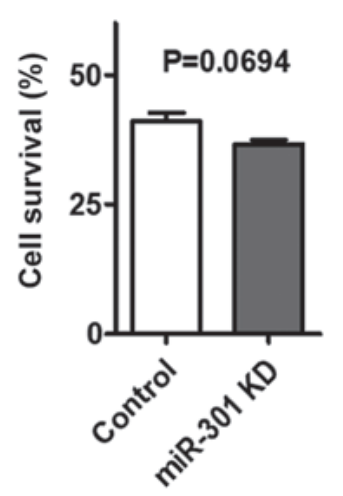

\section{Gemcitabine seneitivity}

Panc-1 GEM-R $(0.2 \mu \mathrm{M})$

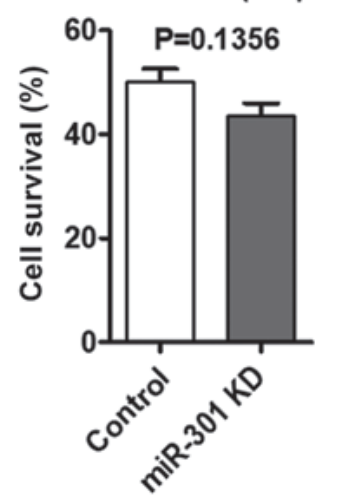

Figure 10. miR-301 inhibition did not affect gemcitabine resistance in GEM-R cells. miR-301 inhibitor was transfected in Capan-2 and Panc-1 GEM-R cells Transfection efficiency was determined using reverse transcription-quantitative polymerase chain reaction analysis, and data are displayed as a relative fold change. Data represent the mean of triplicate samples from three independent experiments. The gemcitabine sensitivity assay demonstrated that miR-301 inhibition did not reduce gemcitabine resistance in either cell line. miR, microRNA; KD, knockdown; GEM-R, gemcitabine-resistant.

with alternative drugs; however, the combined regimens, including oxaliplatin, erlotinib and nab-paclitaxel, have not produced markedly different results in terms of overall survival (38-40). Therefore, it is necessary to identify effective biomarkers for chemosensitivity to gemcitabine and to elucidate the mechanism underlying the development of gemcitabine resistance. Recently, evidence has suggested that a number of miRNAs may affect chemosensitivity to gemcitabine for the treatment of malignant tumors, including miR-301, miR-200b, miR-29a and miR-145 $(13,21,41,42)$. The present study focused on miR-200b and miR-301, as they are known to be involved in the progression of several types of cancers $(13,17,18)$. Since the molecular mechanisms of miR-200b and miR-301 in pancreatic carcinoma in relation to gemcitabine treatment remain unclear, it was hypothesized that the expression levels of miR-200b and miR-301 may serve as surrogate predictors of chemosensitivity and chemoresistance through EMT in pancreatic carcinoma treated with gemcitabine. miR-200b is known to be a tumor suppressor, and serves an important role in the development and progression of malignant tumors. A number of studies have demonstrated that miR-200b expression is reduced in various types of cancer, including pancreatic carcinoma $(18,22)$. Additionally, Gui et al (43) reported that overexpressed miR-200b significantly inhibited cell migration by targeting ZEB1 in pancreatic carcinoma cells. Moreover, it was demonstrated that overexpressed miR-200b increased chemosensitivity to gemcitabine (43). Notably, a recent report revealed that docetaxel chemoresistance is closely associated with the downregulation of miR-200b and the corresponding upregulation of autophagy-associated gene 12 in lung cancer (44). Furthermore, Asakura et al (45) reported that miR-200b is associated with proteasome inhibitor resistance by targeting E-cadherin suppression. Consistently, our previous study also illustrated the same phenomenon of gemcitabine resistance through EMT in pancreatic carcinoma cells (25). Therefore, miR-200b may be associated with the regulation of gemcitabine sensitivity as a tumor suppressor gene. Secondarily, accumulated evidence has revealed that miR-301 expression is upregulated in a number of malignant tumors (46). Furthermore, miR-301 reportedly functions as an oncogene (47). In addition, Shi et al (26) reported that reduced miR-301 expression increased tamoxifen sensitivity 
via targeting of forkhead box F2, BCL2 biding component 3, phosphatase and tensin homolog and collagen type II $\alpha 1$ chain. Moreover, a recent study revealed that miR-301 contributes to the activation of $\mathrm{NF}-\kappa \mathrm{B}$ in pancreatic carcinoma (48). In previous work, it was demonstrated that the miR-301/NF- $\mathrm{B}$ axis critically promotes the upregulation of gemcitabine resistance in pancreatic carcinoma cells (13). Therefore, the present findings indicated that miR-301 may serve an important role as an oncogene.

In the current study, it was identified that miR-200b expression correlated positively with $\mathrm{IC}_{50}$ in six pancreatic carcinoma cell lines, and overexpressed miR-200b affected gemcitabine sensitivity, cell invasiveness, apoptosis and cell proliferation. These results suggested that miR-200b expression may predict gemcitabine sensitivity prior to the introduction of initial chemotherapy. By contrast, it was observed that miR-301 promoted gemcitabine resistance in Capan-2 and Panc-1 cells. Furthermore, it was demonstrated that gemcitabine-resistant cells exhibited increased miR-301 expression in the Capan-2 and Panc-1 cell lines. Capan-2-GEM-R and Panc-1-GEM-R cells exhibited increased $\mathrm{IC}_{50}$ values for gemcitabine compared with their respective control cell lines. By contrast, exogenous miR-301 inhibited apoptosis and enhanced cell invasion, consistent with previous data (13). However, a limitation of the present study is the lack of flow cytometry or data on poly-ADP-ribose polymerase to reinforce the association between miR-301 and apoptosis. Notably, miR-200b and miR-301 affected EMT through CDH1 expression $(13,25)$. However, no morphological alterations were observed in either cell line despite the alteration of $\mathrm{CDH} 1$ expression. Based on these results and the evidence above, the present data suggested that miR-200b and miR-301 may be predictive markers for response to gemcitabine in patients with pancreatic carcinoma. However, the exact molecular mechanisms underlying the ability of miR-200b and miR-301 to regulate chemosensitivity and acquired chemoresistance are unknown, and these mechanisms require further investigation and validation in additional studies in vivo.

In conclusion, the present study provided evidence that miR-200b and elevated miR-301 may be useful biomarkers to predict the chemosensitivity and acquired chemoresistance of pancreatic carcinoma to gemcitabine. The present data also indicated that tailoring treatments according to miR-200b and miR-301 expression in such patients may be considered for the treatment of pancreatic carcinoma in routine clinical practice.

\section{Acknowledgements}

The authors would like to thank Dr Noriko Funamizu (Department of Internal Medicine, Hirose Hospital, Ehime, Japan) for helpful discussions throughout this work.

\section{Funding}

No funding was received.

\section{Availability of data and materials}

The datasets used and/or analyzed during the current study are available from the corresponding author on reasonable request.

\section{Authors' contributions}

NF performed the experimental studies, and drafted and completed the manuscript. CRL and MK participated in the design of the study. KY and YM conceived the project and supervised the research. NF conceived of the study and performed the statistical analysis. All authors read and approved the final manuscript.

\section{Ethics approval and consent to participate}

Not applicable.

\section{Consent for publication}

Not applicable.

\section{Competing interests}

The authors declare that they have no competing interests.

\section{References}

1. Siegel RL, Miller KD and Jemal A: Cancer statistics, 2016. CA Cancer J Clin 66: 7-30, 2016.

2. Oettle H: Progress in the knowledge and treatment of advanced pancreatic cancer: From benchside to bedside. Cancer Treat Rev 40: 1039-1047, 2014.

3. Paulson AS, Tran Cao HS, Tempero MA and Lowy AM: Therapeutic advances in pancreatic cancer. Gastroenterology 144: 1316-1326, 2013

4. Li D, Xie K, Wolff R and Abbruzzese JL: Pancreatic cancer. Lancet 363: 1049-1057, 2004.

5. Bartel DP: MicroRNAs: Genomics, biogenesis, mechanism, and function. Cell 116: 281-297, 2004.

6. Iorio MV and Croce CM: MicroRNA dysregulation in cancer: Diagnostics, monitoring and therapeutics. A comprehensive review. EMBO Mol Med 4: 143-159, 2012.

7. Nikitina EG, Urazova LN and Stegny VN: MicroRNAs and human cancer. Exp Oncol 34: 2-8, 2012

8. Piepoli A, Tavano F, Copetti M, Mazza T, Palumbo O, Panza A, di Mola FF, Pazienza V, Mazzoccoli G, Biscaglia G, et al: Mirna expression profiles identify drivers in colorectal and pancreatic cancers. PLoS One 7: e33663, 2012.

9. Subramani R, Gangwani L, Nandy SB, Arumugam A, Chattopadhyay $\mathrm{M}$ and Lakshmanaswamy R: Emerging roles of microRNAs in pancreatic cancer diagnosis, therapy and prognosis (Review). Int J Oncol 47: 1203-1210, 2015.

10. Li C, Hashimi SM, Good DA, Cao S, Duan W, Plummer PN, Mellick AS and Wei MQ: Apoptosis and microRNA aberrations in cancer. Clin Exp Pharmacol Physiol 39: 739-746, 2012.

11. Wu Y, Xiao Y, Ding X, Zhuo Y, Ren P, Zhou C and Zhou J: A miR-200b/200c/429-binding site polymorphism in the 3 ' untranslated region of the AP- $2 \alpha$ gene is associated with cisplatin resistance. PLoS One 6: e29043, 2011.

12. Garofalo M, Romano G, Di Leva G, Nuovo G, Jeon YJ, Ngankeu A, Sun J, Lovat F, Alder H, Condorelli G, et al: EGFR and MET receptor tyrosine kinase-altered microRNA expression induces tumorigenesis and gefitinib resistance in lung cancers. Nat Med 18: 74-82, 2011.

13. Funamizu N, Lacy CR, Parpart ST, Takai A, Hiyoshi Y and Yanaga K: MicroRNA-301b promotes cell invasiveness through targeting TP63 in pancreatic carcinoma cells. Int J Oncol 44: 725-734, 2014

14. Wang Z, Li Y, Ahmad A, Azmi AS, Kong D, Banerjee S and Sarkar FH: Targeting miRNAs involved in cancer stem cell and EMT regulation: An emerging concept in overcoming drug resistance. Drug Resist Updat 13: 109-118, 2010.

15. Zhang WL, Zhang JH, Wu XZ, Yan T and Lv W: miR-15b promotes epithelial-mesenchymal transition by inhibiting SMURF2 in pancreatic cancer. Int J Oncol 47: 1043-1053, 2015. 
16. Bai Z, Sun J, Wang X, Wang H,Pei H and Zhang Z: MicroRNA-153 is a prognostic marker and inhibits cell migration and invasion by targeting SNAI1 in human pancreatic ductal adenocarcinoma. Oncol Rep 34: 595-602, 2015.

17. Pacurari M, Addison JB, Bondalapati N, Wan YW, Luo D, Qian Y, Castranova V, Ivanov AV and Guo NL: The microRNA-200 family targets multiple non-small cell lung cancer prognostic markers in $\mathrm{H} 1299$ cells and BEAS-2B cells. Int J Oncol 43 : 548-560, 2013

18. Minn YK, Lee DH, Hyung WJ, Kim JE, Choi J, Yang SH, Song H, Lim BJ and Kim SH: MicroRNA-200 family members and ZEB2 are associated with brain metastasis in gastric adenocarcinoma. Int J Oncol 45: 2403-2410, 2014.

19. Shen A, Lin W, Chen Y, Liu L, Chen H, Zhuang Q, Lin J, Sferra TJ and Peng J: Pien Tze Huang inhibits metastasis of human colorectal carcinoma cells via modulation of TGF- $\beta 1 / \mathrm{ZEB}$ miR-200 signaling network. Int J Oncol 46: 685-690, 2015.

20. Li L, Li B, Chen D, Liu L, Huang C, Lu Z, Lun L and Wan X: miR-139 and miR-200c regulate pancreatic cancer endothelial cell migration and angiogenesis. Oncol Rep 34: 51-58, 2015.

21. Li Y, VandenBoom TG II, Kong D, Wang Z, Ali S, Philip PA and Sarkar FH: Up-regulation of miR-200 and let-7 by natural agents leads to the reversal of epithelial-to-mesenchymal transition in gemcitabine-resistant pancreatic cancer cells. Cancer Res 69 6704-6712, 2009.

22. Chang L, Guo F, Huo B, Lv Y, Wang Y and Liu W: Expression and clinical significance of the microRNA-200 family in gastric cancer. Oncol Lett 9: 2317-2324, 2015.

23. Gregory PA, Bert AG, Paterson EL, Barry SC, Tsykin A, Farshid G, Vadas MA, Khew-Goodall Y and Goodall GJ: The miR-200 family and miR-205 regulate epithelial to mesenchymal transition by targeting ZEB1 and SIP1. Nat Cell Biol 10: 593-601, 2008.

24. Sun L, Yao Y,Liu B, Lin Z, Lin L, Yang M, Zhang W, Chen W, Pan C, Liu Q, et al: MiR-200b and miR-15b regulate chemotherapy-induced epithelial-mesenchymal transition in human tongue cancer cells by targeting BMI1. Oncogene 31: 432-445, 2012.

25. Funamizu N, Hu C, Lacy C, Schetter A, Zhang G, He P, Gaedcke J, Ghadimi MB, Ried T, Yfantis HG, et al: Macrophage migration inhibitory factor induces epithelial to mesenchymal transition, enhances tumor aggressiveness and predicts clinical outcome in resected pancreatic ductal adenocarcinoma. Int J Cancer 132: 785-794, 2013.

26. Shi W, Gerster K, Alajez NM, Tsang J, Waldron L, Pintilie M, Hui AB, Sykes J, P'ng C, Miller N, et al: MicroRNA-301 mediates proliferation and invasion in human breast cancer. Cancer Res 71: 2926-2937, 2011.

27. Duxbury MS, Ito H, Zinner MJ, Ashley SW and Whang EE: Inhibition of SRC tyrosine kinase impairs inherent and acquired gemcitabine resistance in human pancreatic adenocarcinoma cells. Clin Cancer Res 10: 2307-2318, 2004.

28. Funamizu N, Kamata Y, Misawa T, Uwagawa T, Lacy CR, Yanaga K, Manome Y: Hydroxyurea decreases gemcitabine resistance in pancreatic carcinoma cells with highly expressed ribonucleotide reductase. Pancreas 41: 107-113, 2012.

29. Livak KJ and Schmittgen TD: Analysis of relative gene expression data using real-time quantitative PCR and the 2(- $\Delta \Delta C(T))$ method. Methods 25: 402-408, 2001.

30. Funamizu N, Okamoto A, Kamata Y, Misawa T, Uwagawa T, Gocho T, Yanaga K and Manome Y: Is the resistance of gemcitabine for pancreatic cancer settled only by overexpression of deoxycytidine kinase? Oncol Rep 23: 471-475, 2010.

31. Funamizu N, Lacy CR, Fujita K, Furukawa K, Misawa T, Yanaga $\mathrm{K}$ and Manome Y: Tetrahydrouridine inhibits cell proliferation through cell cycle regulation regardless of cytidine deaminase expression levels. PLoS One 7: e37424, 2012.

32. Kamada M, Akiyoshi K, Akiyama N, Funamizu N, Watanabe M, Fujioka K, Ikeda K and Manome Y: Cholangiocarcinoma cell line TK may be useful for the pharmacokinetic study of the chemotherapeutic agent gemcitabine. Oncol Rep 32: 829-834, 2014.
33. Funamizu N, Lacy CR, Kamada M, Yanaga K and Manome Y: MicroRNA-203 induces apoptosis by upregulating Puma expression in colon and lung cancer cells. Int $\mathrm{J}$ Oncol 47: 1981-1988, 2015

34. Wang Z, Chen Y, Lin Y, Wang X, Cui X, Zhang Z, Xian G and Qin C: Novel crosstalk between KLF4 and ZEB1 regulates gemcitabine resistance in pancreatic ductal adenocarcinoma. Int J Oncol 51: 1239-1248, 2017.

35. Gillen S, Schuster T, Meyer Zum Büschenfelde C, Friess H and Kleeff J: Preoperative/neoadjuvant therapy in pancreatic cancer: A systematic review and meta-analysis of response and resection percentages. PLoS Med 7: e1000267, 2010.

36. Abou-Alfa GK, Letourneau R, Harker G, Modiano M, Hurwitz H, Tchekmedyian NS, Feit K, Ackerman J, De Jager RL, Eckhardt SG, et al: Randomized phase III study of exatecan and gemcitabine compared with gemcitabine alone in untreated advanced pancreatic cancer. J Clin Oncol 24: 4441-4447, 2006.

37. Burris HA III, Moore MJ, Andersen J, Green MR, Rothenberg ML, Modiano MR, Cripps MC, Portenoy RK, Storniolo AM, Tarassoff $\mathrm{P}$, et al: Improvements in survival and clinical benefit with gemcitabine as first-line therapy for patients with advanced pancreas cancer: A randomized trial. J Clin Oncol 15: 2403-2413, 1997.

38. Louvet C, Labianca R, Hammel P, Lledo G, Zampino MG, André T, Zaniboni A, Ducreux M, Aitini E, Taïeb J, et al; GERCOR; GISCAD: Gemcitabine in combination with oxaliplatin compared with gemcitabine alone in locally advanced or metastatic pancreatic cancer: Results of a GERCOR and GISCAD phase III trial. J Clin Oncol 23: 3509-3516, 2005.

39. Vickers MM, Powell ED, Asmis TR, Jonker DJ, Hilton JF, O'Callaghan CJ, Tu D, Parulekar W and Moore MJ: Comorbidity, age and overall survival in patients with advanced pancreatic cancer - results from NCIC CTG PA.3: A phase III trial of gemcitabine plus erlotinib or placebo. Eur J Cancer 48: 1434-1442, 2012.

40. Von Hoff DD, Ramanathan RK, Borad MJ, Laheru DA, Smith LS, Wood TE, Korn RL, Desai N, Trieu V, Iglesias JL, et al: Gemcitabine plus nab-paclitaxel is an active regimen in patients with advanced pancreatic cancer: A phase I/II trial. J Clin Oncol 29: 4548-4554, 2011

41. Nagano H, Tomimaru Y, Eguchi H, Hama N, Wada H, Kawamoto K, Kobayashi S, Mori M and Doki Y: MicroRNA-29a induces resistance to gemcitabine through the Wnt/ $\beta$-catenin signaling pathway in pancreatic cancer cells. Int J Oncol 43: 1066-1072, 2013

42. Lin Y, Ge X, Wen Y, Shi ZM, Chen QD, Wang M, Liu LZ, Jiang BH and Lu Y: MiRNA-145 increases therapeutic sensibility to gemcitabine treatment of pancreatic adenocarcinoma cells. Oncotarget 7: 70857-70868, 2016.

43. Gui Z, Luo F, Yang Y, Shen C, Li S and Xu J: Oridonin inhibition and $\mathrm{miR} 200 \mathrm{~b} 3 \mathrm{p} / \mathrm{ZEB} 1$ axis in human pancreatic cancer. Int J Oncol 50: 111-120,2017.

44. Pan B, Feng B, Chen Y, Huang G, Wang R, Chen L and Song H: MiR-200b regulates autophagy associated with chemoresistance in human lung adenocarcinoma. Oncotarget 6: 32805-32820, 2015.

45. Asakura T, Yamaguchi N, Ohkawa K and Yoshida K: Proteasome inhibitor-resistant cells cause EMT-induction via suppression of E-cadherin by miR-200 and ZEB1. Int J Oncol 46: 2251-2260, 2015.

46. Lee EJ, Gusev Y, Jiang J, Nuovo GJ, Lerner MR, Frankel WL, Morgan DL, Postier RG, Brackett DJ and Schmittgen TD: Expression profiling identifies microRNA signature in pancreatic cancer. Int J Cancer 120: 1046-1054, 2007.

47. Yang S,He P, Wang J, Schetter A, Tang W, Funamizu N, Yanaga K, Uwagawa T, Satoskar AR, Gaedcke J, et al: A novel MIF signaling pathway drives the malignant character of pancreatic cancer by targeting NR3C2. Cancer Res 76: 3838-3850, 2016.

48. Lu Z, Li Y, Takwi A, Li B, Zhang J, Conklin DJ, Young KH, Martin R and Li Y: miR-301a as an NF- $\mathrm{kB}$ activator in pancreatic cancer cells. EMBO J 30: 57-67, 2011. 